\title{
Radiomics Derived Brain Age Predicts Functional Outcome After Acute Ischemic Stroke
}

\section{Martin Bretzner ( $\nabla$ MBRETZNER@mgh.harvard.edu )}

Massachusetts General Hospital https://orcid.org/0000-0002-7594-4159

\section{Anna Bonkhoff}

Massachusetts General Hospital, Harvard Medical School https://orcid.org/0000-0002-5927-1089

Markus Schirmer

Massachusetts General Hospital https://orcid.org/0000-0001-9561-0239

\section{Sungmin Hong}

J. Philip Kistler Stroke Research Center, Massachusetts General Hospital, Harvard Medical School, Boston

\section{Adrian Dalca}

Computer Science and Artificial Intelligence Lab, Massachusetts Institute of Technology, Boston, USA

Kathleen Donahue

Massachusetts General Hospital

\section{Anne-Katrin Giese}

Massachusetts General Hospital

\section{Mark Etherton}

J. Philip Kistler Stroke Research Center, Massachusetts General Hospital, Harvard Medical School, Boston

\section{Pamela Rist}

Massachusetts General Hospital

\section{Marco Nardin}

J. Philip Kistler Stroke Research Center, Massachusetts General Hospital, Harvard Medical School, Boston

\section{Robert Regenhardt}

Massachusetts General Hospital https://orcid.org/0000-0003-2958-3484

\section{Xavier Leclerc}

Univ. Lille, Inserm, CHU Lille, U1172 - LilNCog - Lille Neuroscience \& Cognition, F-59000 Lille, France

\section{Renaud Lopes}

Univ. Lille, Inserm, CHU Lille, U1172 - LilNCog - Lille Neuroscience \& Cognition, F-59000 Lille, France

\section{Morgan Gautherot}

Univ. Lille, Inserm, CHU Lille, U1172 - LilNCog - Lille Neuroscience \& Cognition, F-59000 Lille, France

\section{Clinton Wang}

Massachusetts Institute of Technology 


\section{Oscar Benavente}

Department of Medicine, Division of Neurology, University of British Columbia, Vancouver, British Columbia, Canada

\section{John Cole}

Department of Neurology, University of Maryland School of Medicine and Veterans Affairs Maryland Health Care System, Baltimore, MD, USA

\section{Amanda Donatti}

School of Medical Sciences, University of Campinas (UNICAMP) and the Brazilian Institute of Neuroscience and Neurotechnology (BRAINN), Campinas, SP, Brazil.

\section{Christoph Griessenauer}

Geisinger

\section{Laura Heitsch}

Division of Emergency Medicine, Washington University School of Medicine, St Louis, MO, USA

\section{Lukas Holmegaard}

Department of Clinical Neuroscience, Institute of Neuroscience and Physiology, Sahlgrenska Academy, University of Gothenburg, Sweden

\section{Katharina Jood}

Institute of Biomedicine, the Sahlgrenska Academy at University of Gothenburg, Gothenburg, Sweden Jordi Jiménez-Conde

Neurology Department, Hospital del Mar - IMIM (Institut Hospital del Mar d'Investigacions Mèdiques)

\section{Steven Kittner}

Department of Neurology, University of Maryland School of Medicine and Veterans Affairs Maryland Health Care System, Baltimore, MD, USA

\section{Robin Lemmens}

Katholieke Universiteit Leuven

\section{Christopher Levi}

School of Medicine and Public Health, University of Newcastle, Newcastle, New South Wales, Australia; Department of Neurology, John Hunter Hospital, Newcastle, NSW, Australia

\section{Patrick McArdle}

Division of Endocrinology, Diabetes and Nutrition, Department of Medicine, University of Maryland School of Medicine, Baltimore, MD, USA

\section{Caitrin McDonough}

University of Florida

\section{James Meschia}

Department of Neurology, Mayo Clinic, Jacksonville, FL, USA https://orcid.org/0000-0002-4475-8142

\section{Chia-Ling Phuah}

Washington University

\section{Arndt Rolfs}

Universitätsmedizin Rostock 


\section{Stefan Ropele}

Medical University of Graz https://orcid.org/0000-0002-5559-768X

\section{Jonathan Rosand}

J. Philip Kistler Stroke Research Center, Massachusetts General Hospital, Harvard Medical School, Boston

\section{Jaume Roquer}

Universitat Autonoma de Barcelona

\section{Tatjana Rundek}

Department of Neurology and Evelyn F. McKnight Brain Institute, Miller School of Medicine, University of Miami, Miami, FL, USA

\section{Ralph Sacco}

Department of Neurology and Evelyn F. McKnight Brain Institute, Miller School of Medicine, University of Miami, Miami, FL, USA

\section{Reinhold Schmidt}

Medical University of Graz

\section{Pankaj Sharma}

Institute of Cardiovascular Research, Royal Holloway University of London (ICR2UL), Egham, UK St Peter's and Ashford Hospitals, UK

\section{Agnieszka Slowik}

Jagiellonian University

\section{Alessandro Viana Oliveira e Sousa}

University of Campinas

\section{Tara Stanne}

Institute of Biomedicine, the Sahlgrenska Academy at University of Gothenburg, Gothenburg, Sweden

\section{Daniel Strbian}

Division of Neurocritical Care \& Emergency Neurology, Department of Neurology, Helsinki University

Central Hospital, Helsinki, Finland https://orcid.org/0000-0001-9095-2344

\section{Turgut Tatlisumak}

Department of Clinical Neurosciences/Neurology, Institute of Neuroscience and Physiology, Sahlgrenska Academy at University of Gothenburg, Gothenburg

\section{Vincent Thijs}

Stroke Division, Florey Institute of Neuroscience and Mental Health, Heidelberg, Australia and Department of Neurology, Austin Health, Heidelberg, Australia

\section{Achala Vagal}

Department of Radiology, University of Cincinnati College of Medicine, Cincinnati, $\mathrm{OH}$, USA

\section{Johan Wasselius}

Department of Clinical Sciences Lund, Radiology, Lund University, Lund,

\section{Daniel Woo}

University of Cincinnati College of Medicine 


\section{Ona Wu}

Massachusetts General Hospital

\section{Ramin Zand}

Department of Neurology, Geisinger, Danville, PA, USA

\section{Bradford B. Worrall}

University of Virginia

Jane Maguire

University of Technology Sydney, Sydney, Australia

\section{Arne Lindgren}

Department of Neurology, Skåne University Hospital, Lund, Sweden;

\section{Christina Jern}

Department of Laboratory Medicine, Institute of Biomedicine, the Sahlgrenska Academy, University of Gothenburg, Gothenburg, Sweden

\section{Polina Golland}

Massachusetts Institute of Technology

\section{Grégory Kuchcinski}

Univ. Lille, Inserm, CHU Lille, U1172 - LilNCog - Lille Neuroscience \& Cognition, F-59000 Lille, France

\section{Natalia Rost}

Massachusetts General Hospital

\section{Article}

Keywords: Stroke, Radiomics, Prognosis, Brain Health, brain age

Posted Date: September 23rd, 2021

DOl: https://doi.org/10.21203/rs.3.rs-923769/v1

License: (c) (1) This work is licensed under a Creative Commons Attribution 4.0 International License. Read Full License 


\section{Abstract}

While chronological age is one of the most influential determinants of post-stroke outcomes, little is known of the impact of neuroimaging-derived biological brain age. We here first examine whether radiomics analysis of the texture of brain T2-FLAIR MRI images can be used to predict brain age in stroke patients. We then assess the clinical determinants of accelerated brain aging and, finally, its impact on post-stroke functional outcomes. Leveraging a multisite cohort of 4,163 ischemic stroke patients, we show that older-appearing patients have more hypertension, diabetes mellitus, prior strokes, and smoking history and are more likely to develop worse post-stroke outcomes than their younger-appearing counterparts. Our results strengthen the importance of preventive medicine for maintaining brain health in stroke patients as they age and suggest a novel methodology to capture previously undescribed prognostic information available on commonly acquired MRI sequences during routine stroke care.

\section{Introduction}

While chronological age is one of the most influential determinants of post-stroke outcomes ${ }^{1}$, little is known about the impact of neuroimaging-derived brain age, a recently developed biomarker of personalized brain health. ${ }^{2}$ Stroke has devastating effects on both functional and cognitive outcomes, ${ }^{3}$ however, the resilience of an individual to ischemic insults might vary depending on the status of the underlying brain. In fact, "older" appearing brains on MRI, defined by a higher neuroimaging-predicted brain age rather than chronological age, have been associated with diverse diseases, risk factors, lifestyles, and cognitive performances. ${ }^{4,5}$ In contrast, patients with "younger" appearing brains are thought to undergo a healthier aging process with better brain maintenance, featuring a decreased pathological age-related structural degeneration. ${ }^{6}$ While a variety of studies focused on brain age of patients suffering from neurodegenerative or psychiatric diseases ${ }^{2}$, the aging process of stroke patient brains and its impact on stroke outcomes remain largely undescribed.

Although chronological time passes at the same speed for everyone, individuals age at different paces. Thus, individuals can be more sensitive, or, on the contrary, more resilient, to the effects of biological aging. Quantifying the deviation from an expected brain aging distribution could prove relevant for assessing brain health and health prognoses, especially in diseases heavily influenced by age, such as stroke. Some authors showed that the brains of stroke survivors appeared "older" than those of agematched healthy controls, highlighting the detrimental impact of the ischemic insult on the aging trajectory of the brain. ${ }^{7,8}$ Moreover, other authors found an association between a higher brain age and a greater risk of stroke, trapping stroke survivors in a vicious circle. ${ }^{9}$ However, the clinical determinants of brain age are currently unknown, warranting further imaging studies in stroke populations to identify potentially preventable risk factors.

Translation of the brain age biomarker to clinical care remains challenging. Indeed, most of the available neuroimaging brain age prediction methods include spatial registration to an anatomical template and 
cortical parcellation, which requires high-quality research-grade imaging. ${ }^{2,4,10}$ Moreover, T1-weighted imaging is predominantly used in current brain age prediction pipelines but this sequence is uncommonly acquired during acute stroke MRI imaging workup. Therefore, there is a need for frameworks compatible with clinical care settings, especially in time-sensitive diseases such as stroke, featuring T2-FLAIR given its common utilization in both the acute phase and during follow-up. Radiomics, an emerging image quantifying technology, could represent a potential solution as they require little computational power and can be applied to any digitalized medical imaging. ${ }^{11}$ Radiomics could therefore leverage clinical T2FLAIR images for brain health and prognosis assessment for stroke populations.

In this large retrospective imaging study of ischemic stroke patients, we aimed to: 1) assess performances of brain T2-FLAIR MRI radiomics to predict brain age from clinical imaging, 2) understand the clinical determinants of brain age, and 3 ) study its relevance to post-stroke outcome. We hypothesized that stroke patients with a higher radiomics-predicted brain age than their chronological age would have more cardiovascular risk factors and worse post-stroke functional outcomes, especially as observed in minor strokes.

\section{Results}

\section{Population}

All included patients had suffered an ischemic stroke. Population demographics are shown in Table 1. There were $42 \%$ females, mean age was 62.8 (standard deviation 15.0) years. Admission NIHSS scores and follow-up mRS scores were available for 2,234 and 1,871 patients, respectively. Exhaustive ordinal mRS scale (0-6) data were available for 871 patients. Median NIHSS was 3 (IQR: 1-6), good functional outcome was achieved by $72.5 \%$ of patients. 
Table 1

Demographic and clinical characteristics of the study population $(n=4,163)$

\begin{tabular}{|lll|}
\hline Age & Mean (SD) & $\mathbf{6 2 . 8 ( 1 5 . 0 )}$ \\
\hline Female & $\mathrm{n}(\%)$ & $1,748(42.0 \%)$ \\
\hline Hypertension & $\mathrm{n}(\%)$ & $2,825(67.9 \%)$ \\
\hline Diabetes mellitus & $\mathrm{n}(\%)$ & $687(16.5 \%)$ \\
\hline Atrial fibrillation & $\mathrm{n}(\%)$ & $595(14.3 \%)$ \\
\hline Coronary artery disease & $\mathrm{n}(\%)$ & $772(18.5 \%)$ \\
\hline History of smoking & $\mathrm{n}(\%)$ & $1,331(32.0 \%)$ \\
\hline Prior stroke & $\mathrm{n}(\%)$ & $539(12.9 \%)$ \\
\hline WMH volume & Median (IQR) & $4.2 \mathrm{ml}(1.4-11.2)$ \\
\hline NIHSS at baseline* & Median (IQR) & $3(1-6)$ \\
\hline Good functional outcome at 60-180 days* & $\mathrm{n}(\%)$ & $1,356(72.5 \%)$ \\
\hline *NIHSS was available for 2,234 patients. & & \\
\hline **Dichotomized functional outcome (mRS 2 vs. mRS >2) was available for 1,871 patients. \\
\hline SD, standard deviation; NIHSS, NIH Stroke Scale; IQR, interquartile range. \\
\hline diomics brain age predictions and Relative Brain Age
\end{tabular}

The mean predicted brain age was 62.8 years with a mean absolute error (MAE) of 6.9 years. Pearson correlation and coefficient of determination between predicted brain age and chronological age were $r=$ $0.81 p<0.001$, and $R^{2}=0.65$ respectively (Fig. 1). Prediction performances using only radiomics describing the shape and size of the brain parenchyma were lower: $r=0.66 p<0.001, R^{2}=0.45$, mean predicted brain age: 62.8 years, and $M A E=9.0$ years.

Legend: Scatter plots of the T2-FLAIR radiomics (A) predicted brain age and (B) relative brain age (RBA) per chronological age. Patients were colored in red if they had a positive RBA and thus a brain that appeared 'older' to their age-matched peers, or in blue if they had a negative RBA and a 'younger' looking brain. (C) T2-FLAIR axial image of a patient with a positive RBA: predicted brain age $=88$, chronological age $=46, \mathrm{RBA}=36.2$; this patient's brain exhibits multiple cortical and subcortical sequelae, moderate to severe parenchymal atrophy with enlarged ventricles and sulci, and confluent white matter hyperintensities, which extents are unexpectedly large for a 46-year-old patient. (D) T2-FLAIR axial image of a patient with a negative RBA: predicted brain age $=43$, chronological age $=92, \mathrm{RBA}=-38.6$; notwithstanding the left middle cerebral artery lesion, this patient's brain trophicity is maintained, the cortex and the deep gray nuclei are sharply defined, overall describing a healthy brain for this 92 -year-old patient. 


\section{Clinical phenotype and brain aging}

In univariable analysis, patients with $\mathrm{HTN}, \mathrm{DM}, \mathrm{AF}, \mathrm{CAD}$, history of smoking, and a history of prior stroke had a significantly higher RBA. RBA did not differ between males and females (Table 2). There was no significant correlation between chronological age and RBA: $r=0.03, p=0.145$.

Table 2

Comparison of patients' Relative Brain Age (RBA) by clinical phenotype $(n=4,163)$

\begin{tabular}{|lccc|}
\hline \multicolumn{1}{|c}{ No } & Yes & p-value \\
\hline Female & $0.02 \pm 7.16$ & $-0.02 \pm 7.23$ & $\mathrm{p}=0.863$ \\
\hline Hypertension & $-0.61 \pm 7.45$ & $0.29 \pm 7.05$ & $\mathrm{p}<0.001$ \\
\hline Diabetes mellitus & $-0.18 \pm 7.16$ & $0.92 \pm 7.03$ & $\mathrm{p}<0.001$ \\
\hline Atrial fibrillation & $-0.10 \pm 7.14$ & $0.60 \pm 7.48$ & $\mathrm{p}=0.034$ \\
\hline Coronaropathy & $0.13 \pm 7.29$ & $0.58 \pm 6.74$ & $\mathrm{p}=0.014$ \\
\hline History of smoking & $-0.15 \pm 7.07$ & $0.33 \pm 7.43$ & $\mathrm{p}=0.045$ \\
\hline $\begin{array}{l}\text { Prior stroke } \\
\text { Legend: Two-sided t-test, alpha = 5\%. Patients with positive RBA have “older" looking brains, whereas } \\
\text { patients with negative RBA have "younger" looking brains. }\end{array}$ & \\
\hline $\begin{array}{l}\text { In multiple regression analyses, RBA was higher in patients with HTN, DM, history of smoking, and } \\
\text { prior stroke (Table 3). }\end{array}$ & $-0.42 \pm 7.07$ & $\mathrm{p}<0.001$ \\
\hline
\end{tabular}

Table 3

Multivariable linear regression of the clinical predictors of relative brain age $(n=4,163)$

\begin{tabular}{|lllll|}
\hline & Coefficient & 95\% Cl & p-value \\
\hline Hypertension & & Lower & Upper \\
\hline Diabetes mellitus & 0.5615 & 0.085 & 1.038 & 0.021 \\
\hline Atrial Fibrillation & 0.9098 & 0.318 & 1.502 & 0.003 \\
\hline Coronary artery disease & 0.4911 & -0.133 & 1.115 & 0.123 \\
\hline History of Smoking & 0.1824 & -0.387 & 0.752 & 0.530 \\
\hline Prior Stroke & 0.5367 & 0.07 & 1.003 & 0.024 \\
\hline Legend: Two-sided t-test, alpha $=5 \%$. A positive coefficient implies older-looking brains. \\
\hline
\end{tabular}

Relative Brain Age and post-stroke functional outcome 
Patients who achieved a good functional outcome had a significantly lower $R B A(-0.44$ versus $1.45, p<$ 0.001 , Fig. 2) and therefore a younger-looking brain than their age-matched peers.

Among the evaluated predictors of post-stroke outcome, the automated cross-validated recursive feature elimination process selected four clinical variables to enter the final logistic regression model: age, baseline NIHSS, prior stroke, and RBA. Results of the logistic regression of good functional outcomes are presented in Table 4. Briefly, in multivariable analysis, a greater chronological age, positive RBA, higher baseline NIHSS score, or presence of a history of prior stroke were independently associated with worse post-stroke outcomes (respective adjusted Odds-Ratios for good outcome: $0.58,0.76,0.48,0.55$; all $p$ values< 0.001$)$.

Table 4

Logistic regression of good functional outcome

\begin{tabular}{|c|c|c|c|c|c|c|c|}
\hline \multirow{3}{*}{ Variables } & \multicolumn{3}{|c|}{ Unstandardized aOR } & \multicolumn{4}{|c|}{ Standardized aOR } \\
\hline & \multirow[t]{2}{*}{$\mathrm{aOR}$} & \multicolumn{2}{|c|}{$95 \% \mathrm{Cl}$ for aOR } & \multirow[t]{2}{*}{$\mathrm{aOR}$} & \multicolumn{2}{|c|}{$95 \% \mathrm{Cl}$ for $\mathrm{aOR}$} & \multirow[t]{2}{*}{$\mathrm{p}$-value } \\
\hline & & Lower & Upper & & Lower & Upper & \\
\hline Age & 0.96 & 0.95 & 0.97 & 0.58 & 0.51 & 0.65 & $<0.001$ \\
\hline NIHSS & 0.87 & 0.85 & 0.89 & 0.48 & 0.43 & 0.54 & $<0.001$ \\
\hline Prior stroke & 0.55 & 0.41 & 0.74 & 0.55 & 0.41 & 0.74 & $<0.001$ \\
\hline RBA & 0.96 & 0.95 & 0.98 & 0.76 & 0.68 & 0.86 & $<0.001$ \\
\hline \multicolumn{8}{|c|}{ Legend: NIHSS NIH Stroke Scale, RBA Relative Brain Age, aOR Adjusted Odds-Ratio. } \\
\hline
\end{tabular}

Legend: Q1-Q4 quartiles of relative brain age, mRS modified Rankin Scale. Q1-Q2 represents patients with lower RBA and thus younger-looking brains, whereas Q3-Q4 represents older-looking brains.

Multivariable effect sizes of RBA on the dichotomized post-stroke outcome by the rank of baseline NIHSS are shown in Fig. 4. After adjustment for covariates (age, NIHSS, history of prior stroke), the detrimental effect sizes of RBA on achieving a good functional outcome were maximal for $N I H S S=0$ (aOR $=0.61, n=$ 295) and decreased until NIHSS $\leq 9(\mathrm{aOR}=0.75, \mathrm{n}=1,642)$.

Legend: RBA relative brain age, NIHSS NIH stroke scale, aOR adjusted Odds-Ratio. RBA had a detrimental impact on achieving a good functional outcome especially in patients with low NIHSS.

\section{Discussion}

By leveraging a large ischemic stroke clinical imaging cohort, we successfully used brain MRI T2-FLAIR radiomics to predict brain age and derived RBA, a biomarker describing patients' brain health relative to 
their peers. "Older" appearing brains were associated with cardiovascular risk factors, highlighting their detrimental impact on brain health. Finally, we showed that high RBA had a negative impact on functional outcomes in general. This effect was especially pronounced in patients presenting with a lower stroke severity.

Chronological age quantifies the length of time a person has lived but is unlikely to impact stroke prognosis directly. It may best serve as a surrogate marker for age-related cerebral parenchymal alterations. In some patients, such deleterious alterations accumulate more rapidly, or more slowly, than the expected pace. Our results suggest that quantifying the deviation from expected brain age in stroke patients can be relevant for assessing brain health and prognostication. Indeed, in our cohort, patients who did not achieve a favorable outcome had brains that appeared older on T2-FLAIR compared to their chronological age-matched peers. Moreover, our results show that RBA impacted stroke outcomes, independently from chronological age, NIHSS, and history of prior stroke. Indeed, patients with an olderappearing brain were more likely to develop an unfavorable post-stroke outcome at any given age. This finding indicates that radiomics-derived RBA could assess the brain health of stroke patients and quantify the resilience of brains in a way that chronological age cannot. In previous analyses of randomized control trials, chronological age-defined elderly patients ( $>80$ years) were identified as a subgroup that benefitted more from endovascular recanalization than their younger counterparts, with the hypothesis that they had less brain reserve to withstand ischemia. ${ }^{20,21}$ Future studies could evaluate if patients with "elderly" appearing brains would benefit more from recanalization ${ }^{22}$ and assess if brain age imaging biomarkers can help to identify candidates for reperfusion therapies.

Individual lifestyles, genetics, and environment can also set a different course for brain aging. ${ }^{4,10} \mathrm{We}$ found that high RBA was associated with HTN, DM, history of smoking, in line with results based on large community-dwelling cohorts like the UK Biobank and Whitehall II. ${ }^{4,5,9,10}$ This adds to the body of evidence that cardiovascular health and brain health are intertwined and stresses the importance of preventative medicine. ${ }^{23}$ Our results also showed that a history of prior stroke was the most influential clinical factor impacting RBA, with an effect size 3-fold larger than other clinical variables. The second most detrimental clinical trait for brain aging in our sample was diabetes, which also emerged as a significant condition accelerating brain aging in previous work. ${ }^{24}$ Identifying potentially modifiable factors impacting brain health yields relevance for prevention interventions. ${ }^{25}$ RBA and brain age could furthermore be used as follow-up brain health biomarkers.

Several sources of data and methods have been developed to calculate brain age. The majority of them leveraged either voxel-wise information ${ }^{2}$, grey and white matter volumes ${ }^{8}$, or template parcellated volumes ${ }^{17}$ data to predict brain age. To our knowledge, radiomics were never assessed as an approach to predict brain age. While in previous work brain age is mostly predicted by volumetric information, radiomics description encompass information that goes beyond volume, characterizing the shape and the texture of an image; consequently, radiomics can potentially capture more information. Analyses of T2-FLAIR images using radiomics is especially relevant in stroke patients, as this sequence reflects both 
higher age and cerebral burden of diseases, as previously shown in previous work. ${ }^{26}$ We did not have access to T1-weighted imaging and consequently, we could not compare previous brain age methodologies with our method. Moreover, most published studies have trained their models on healthy brains, which are more likely to have a uniform aging distribution. This aspect might be reflected by the greater errors of our predictions than published literature, which ranges in adult cohorts from around 2.5 years, for recent complex deep-learning-based methods, ${ }^{27}$ to between 4.3 and 13.5 years for more traditional methods. ${ }^{2}$ There is a trend in brain age predictions literature to incorporate more and more complex and multimodal data, such as diffusion tensor imaging or functional MRI, and process it within high-end deep-learning frameworks. ${ }^{10,28,29}$ We deliberately chose to tackle the challenge of leveraging clinical imaging and a lighter methodological framework, with the idea of developing models that are more interpretable and may have a better chance of translating brain age biomarkers to routine clinical care.

This work has several limitations. First, we could not benchmark our method against other published methods like BrainAGE or Brain age delta2,17, as we did not have T1-weighted images, further work could evaluate our method on reference datasets if they include T2-FLAIR imaging. Second, we could not explore the relationship between T2-FLAIR radiomics derived brain age biomarkers and more detailed outcome metrics such as cognitive or language outcomes. Future studies could indeed study the relationship between radiomics brain age and cognitive reserve in stroke patients. Lastly, our dataset included patients from 2003 to 2011, who might not be representative of patients treated in modern stroke care settings, further work could evaluate our method on modern cohorts of patients treated with up-to-date reperfusion therapies.

To conclude, in this cohort study of 4,163 ischemic stroke patients, using radiomics extracted from clinically acquired T2-FLAIR images, we derived relative brain age, a chronological-age-independent biomarker describing individual biological brain age. A higher relative brain age was linked to the presence of cardiovascular risk factors and worse post-stroke outcomes. Therefore, this newly radiomicsderived relative brain age may capture previously unidentified prognostic information.

\section{Methods}

\section{Participants}

We reviewed all stroke patients' neuroimaging data included in a large international multisite collaborative effort: the MRI-GENetics Interface Exploration (MRI-GENIE) study. Sites shared clinical, MRI imaging, and genetic data. Both study design, data collection protocols, and populations have been previously published. ${ }^{12,13}$

\section{Ethics}


All participants or health care proxy provided signed informed consent. The MRI-GENIE project has been approved by the MGH Institutional Review Board (IRB, Protocol \#: 2001P001186 and Protocol \#: 2003P000836), as well as the ethics boards of the collaborating institutions.

\section{Data Collection and neuroimaging pre-processing}

We reviewed a total of 4,163 patients across 17 different sites, for which cardiovascular risk factor phenotypes, T2-FLAIR imaging, and successful brain and ventricles segmentations were available. ${ }^{14}$ Demographic and cardiovascular phenotypes included age, sex, hypertension (HTN), history of smoking, diabetes mellitus (DM), atrial fibrillation (AF), and a history of prior stroke. Acute stroke severity was measured with the National Institutes of Health Stroke Scale (NIHSS) scale. Functional outcome was measured with the modified Rankin scale (mRS) at 60-180 days post-stroke.

Axial T2-FLAIR images were acquired between 2003 and 2011 within 48 hours of the hospital admission. Mean in-plane resolution was $0.7 \mathrm{~mm}$ (range: $0.3 \mathrm{~mm}-1.0 \mathrm{~mm}$ ) and mean through-plane resolution was $6.2 \mathrm{~mm}$ (range: $3.0 \mathrm{~mm}-30.0 \mathrm{~mm}$ ). Total brain, ventricle, and white matter hyperintensities (WMH) were automatically segmented using dedicated state-of-the-art deep-learning frameworks. ${ }^{14,15}$ T2-FLAIR images intensities were normalized. ${ }^{14}$ We computed parenchymal masks by subtracting the ventricle masks from the total brain masks. Then we performed a morphological opening operation with a $3 \times 3$ kernel to each axial slice to prevent any segmentation noise from perturbating radiomics extraction.

\section{Radiomic feature extraction}

Radiomic features were extracted using the open-source toolbox PyRadiomics V2.2.0 from brain parenchyma on T2-FLAIR. ${ }^{16}$ Briefly, 760 features were extracted describing the shape, histogram, and texture of the brains. The list of the extraction parameters can be found at https://github.com/MBretzner/Brain-Age-Radiomics (uploaded upon acceptance).

\section{Chronological Age, Brain Age, and Relative Brain Age}

Radiomics-derived predictions of patients' chronological ages were performed by an ElasticNet linear regression model in a 5-fold nested stratified cross-validation scheme. First, the whole dataset was split into five equivalent training and test samples $(80 / 20 \%)$ in order to produce one single out-of-trainingsample age prediction for every patient. Then, for each of the five train-test splits, feature selection was performed on the training set by an ElasticNet linear regression model in a 3-fold cross-validation scheme (inner loop). Selected features were subsequently entered into another ElasticNet model that was fitted on the entire training set where its L1 and L2 hyperparameters were optimized, and then finally tested on the unseen test set (outer loop). Radiomics-based predicted ages are subsequently referred to as "brain age". Prediction performances were evaluated with Pearson correlation $(r)$ and coefficient of determination $\left(R^{2}\right)$.

To evaluate the specific added value of brain age to chronological age, we calculated the residuals of predictions (brain age - chronological age). However, these residuals are known to be negatively 
correlated with chronological age because of a regression dilution bias induced by the accumulation of random measurement errors, which can be encountered in radiomics analyses. ${ }^{17}$ An established solution suggested by Ning and colleagues ${ }^{4}$ is to calculate Relative Brain Age (RBA) by regressing out any correlation with chronological age as follow:

$$
R B A=\text { PredictedAge - Expected (PredictedAge } \mid \text { ChronologicalAge) }
$$

The expected predicted age as a function of chronological age is obtained by fitting a linear regression model with chronological age as an input and the predicted age as a response variable. RBA represents the appearance of an individual brain in comparison with chronological age-matched peers within the cohort: a higher brain age at a given chronological age will have a positive RBA and will reflect an older looking brain, whereas a younger appearing brain on neuroimaging will have a negative RBA.

To evaluate the added value of detailed textural brain information on top of simple volumetric information of the brain mask, an ancillary analysis was performed: we here predicted brain age using only those radiomics features describing the size and the shape of the parenchyma but not the more detailed texture. This analysis was performed to produce brain age biomarkers based on the cerebral parenchymal volumetric data.

\section{Identifying clinical determinants of accelerated aging}

To quantify the impact of patients' clinical phenotypes on their brain, RBA were compared using a twosided t-test for each categorical clinical variable (sex, HTN, DM, AF, CAD, history of smoking, history of prior stroke), and via Pearson correlation for continuous variables (age) (level of significance: $p<0.05$ ). Variables for which RBA significantly differed in univariate analyses were then entered into a multiple linear regression model of RBA.

\section{Evaluating the impact of accelerated brain aging on post- stroke functional outcome}

Good functional outcome was defined as a mRS $\leq 2$ at follow-up. Comparison of patients' RBA by dichotomized outcome groups was done using a two-sided t-test. To compare the effect of RBA on functional outcome with traditional variables, multiple logistic regression of dichotomized functional outcome was built. Feature selection for this model was performed using a five-fold cross-validated recursive feature elimination process. Candidate predictors were age, sex, HTN, DM, AF, CAD, history of smoking, prior stroke, brain volume, WMH volume, NIHSS at index stroke, and RBA. The final model's coefficients were estimated, and odds-ratio were calculated. To further evaluate the impact of RBA beyond dichotomized functional outcome (good vs. bad), full-scale mRS shifts (0-6) were examined by quartiles of RBA.

In ancillary analyses, we investigated our secondary hypothesis that RBA would only be impactful on functional outcomes in minor strokes, as severe strokes may lead to unfavorable outcomes regardless of the underlying brain status. To explore this hypothesis, we studied the effect sizes of RBA on 
dichotomized outcome groups by incrementally adding patients by the rank of NIHSS $(0,0-1,0-2,0-3$, etc.) and estimated the effect sizes by calculating the standardized adjusted odds-ratios of multiple logistic regression models built with the variables previously identified as significantly associated with good functional outcome.

\section{Code availability}

Radiomic features extraction, feature selection, and machine learning analyses were performed in python 3.7.6 using the toolbox scikit-learn and pyradiomics. ${ }^{16,18}$ Logistic regression coefficients estimations were performed using statsmodels. ${ }^{19}$

\section{Data availability}

Upon reasonable request to the corresponding author and pending approval from local IRBs, data will be made available to replicate the results presented in this manuscript.

\section{Declarations}

\section{Funding}

The MRI-GENIE study was funded by NIH NINDS (R01NS086905, NSR PI). M.B was supported by the ISITE-ULNE Fundation, the Société Française de Neuroradiologie, the Société Française de Radiologie, the Thérèse and René Planiol Fundation. A.K.B. is supported by a MGH ECOR Fund for Medical Discovery (FMD) Clinical Research Fellowship Award. MRE is supported by the American Academy of Neurology and MGH Executive Council on Research. PMR is supported by NIH K01 HL128791. TT was supported by the Helsinki University Central Hospital, Sigrid Juselius Foundation, Sahlgrenska University Hospital, and University of Gothenburg. A.G.L was supported by the Swedish Research Council (2019-01757), CaNVAS project funded by NIH (US) The Swedish Government (under the Avtal om Läkarutbildning och Medicinsk Forskning, ALF), The Swedish Heart and Lung Foundation, Region Skåne, Lund University, Skåne University Hospital, Sparbanksstiftelsen Färs och Frosta, Fremasons Lodge of Instruction Eos in Lund. C.J. was supported by the Swedish Research Council (2018-02543); the Swedish Heart and Lung Foundation (20190203); and the Swedish state under the agreement between the Swedish government and the county councils, the ALF agreement (ALFGBG-720081) PL is supported by NIH NIBIB NAC P41EB015902; The funders had no role in design and conduct of the study; collection, management, analysis, and interpretation of the data; preparation, review, or approval of the manuscript; and decision to submit the manuscript for publication.

\section{Contributions}

Conception and design of the study: MB, AKB, MDS, SH, MRE, RM, CW, RWR, XL, RL, GK, NSR; Acquisition and analysis of data: MB, AKB, MDS, SH, DAV, KD, AKG, MRE, MN, ORB, JWC, AD, CJG, LH, LH, JKJ, JJC, 
SJK, RL, CRL, PFM, CWM, JFM, CLP, AR, SR, JR, JR, TJ, RLS, RS, PS, AS AS, TMS, DS, TT, VT, AV, JWC, DW, OW, RZ, BBW, JM, AL, CJ, PG, GK, NSR; Drafting a significant portion of the manuscript or figures: MB, $A K B, S H, M D S, M R E, R W R, X L, R L, G K, N S R$

\section{Competing interests}

M.E. has received personal fees for consulting from Astra Zeneca and WorldCare Clinical Group. C.G. has received consulting honoraria from Microvention and Stryker and research funding from Medtronic and Penumbra. A.V. has received research funding from Cerenovus. A.G.L. has received personal fees from Bayer, Astra Zeneca, BMS Pfizer, and Portola. N.S.R. has received compensation as scientific advisory consultant from Omniox, Sanofi Genzyme and AbbVie Inc.

\section{Abbreviations}

$\mathrm{AF}$

Atrial Fibrillation

$\mathrm{aOR}$

adjusted Odds-Ratio

CAD

Coronary artery disease

DM

Diabetes mellitus

HTN

Hypertension

IQR

Interquartile range

MAE

Mean absolue error

mRS

modified Rankin Scale

NIHSS

$\mathrm{NIH}$ Stroke Scale

RBA

Relative Brain Age

SD

Standard deviation

WMH

White matter hyperintensities

\section{References}


1. Drozdowska BA, Singh S, Quinn TJ. Thinking About the Future: A Review of Prognostic Scales Used in Acute Stroke. Front Neurol. 2019;10:274-274. doi:10.3389/fneur.2019.00274

2. Franke K, Gaser C. Ten Years of BrainAGE as a Neuroimaging Biomarker of Brain Aging: What Insights Have We Gained? Front Neurol. 2019;10:789. doi:10.3389/fneur.2019.00789

3. Hénon H, Pasquier F, Leys D. Poststroke Dementia. Cerebrovascular diseases (Basel, Switzerland). 2006;22:61-70. doi:10.1159/000092923

4. Ning K, Zhao L, Matloff W, Sun F, Toga AW. Association of relative brain age with tobacco smoking, alcohol consumption, and genetic variants. Scientific Reports. 2020;10(1):10. doi:10.1038/s41598019-56089-4

5. Kolbeinsson A, Filippi S, Panagakis Y, et al. Accelerated MRI-predicted brain ageing and its associations with cardiometabolic and brain disorders. Scientific Reports. 2020;10(1):19940. doi:10.1038/s41598-020-76518-z

6. Alzheimer's \& Dementia. 2020;16(9):1305-1311. doi:10.1016/j.jalz.2018.07.219

7. Richard G, Kolskår K, Ulrichsen KM, et al. Brain age prediction in stroke patients: Highly reliable but limited sensitivity to cognitive performance and response to cognitive training. Neurolmage: Clinical. 2020;25:102159. doi:10.1016/j.nicl.2019.102159

8. Egorova N, Liem F, Hachinski V, Brodtmann A. Predicted Brain Age After Stroke. Front Aging Neurosci. 2019;11:348-348. doi:10.3389/fnagi.2019.00348

9. de Lange A-MG, Anatürk M, Suri S, et al. Multimodal brain-age prediction and cardiovascular risk: The Whitehall II MRI sub-study. Neuroimage. 2020;222:117292-117292.

doi:10.1016/j.neuroimage.2020.117292

10. Cole JH. Multimodality neuroimaging brain-age in UK biobank: relationship to biomedical, lifestyle, and cognitive factors. Neurobiol Aging. 2020;92:34-42. doi:10.1016/j.neurobiolaging.2020.03.014

11. Lambin P, Leijenaar RTH, Deist TM, et al. Radiomics: the bridge between medical imaging and personalized medicine. Nat Rev Clin Oncol. 2017;14(12):749-762. doi:10.1038/nrclinonc.2017.141

12. Giese A-K, Schirmer MD, Dalca AV, et al. White matter hyperintensity burden in acute stroke patients differs by ischemic stroke subtype. Neurology. 2020;95(1):e79-e88.

doi:10.1212/WNL.0000000000009728

13. Bonkhoff AK, Schirmer MD, Bretzner M, et al. Outcome after acute ischemic stroke is linked to sexspecific lesion patterns. Nat Commun. 2021;12(1):3289. doi:10.1038/s41467-021-23492-3

14. Schirmer MD, Dalca AV, Sridharan R, et al. White matter hyperintensity quantification in large-scale clinical acute ischemic stroke cohorts - The MRI-GENIE study. Neurolmage: Clinical. 2019;23:101884. doi:10.1016/j.nicl.2019.101884

15. Dubost F, Bruijne $M$ de, Nardin M, et al. Multi-atlas image registration of clinical data with automated quality assessment using ventricle segmentation. Med Image Anal. 2020;63:101698. doi:10.1016/j.media.2020.101698 
16. Griethuysen JJM van, Fedorov A, Parmar C, et al. Computational Radiomics System to Decode the Radiographic Phenotype. Cancer Res. 2017;77(21):e104-e107. doi:10.1158/0008-5472.CAN-17-0339

17. Smith SM, Vidaurre D, Alfaro-Almagro F, Nichols TE, Miller KL. Estimation of brain age delta from brain imaging. Neuroimage. 2019;200:528-539. doi:10.1016/j.neuroimage.2019.06.017

18. Pedregosa F, Varoquaux G, Gramfort A, et al. Scikit-learn: Machine Learning in Python. J Mach Learn Res. 2011;12(null):2825-2830.

19. Seabold S, Perktold J. Statsmodels: Econometric and Statistical Modeling with Python. In: Walt S van der, Millman J, eds. Proceedings of the 9th Python in Science Conference.; 2010:92-96. doi:10.25080/Majora-92bf1922-011

20. Groot AE, Treurniet KM, Jansen IGH, et al. Endovascular treatment in older adults with acute ischemic stroke in the MR CLEAN Registry. Neurology. 2020;95(2):e131-e139.

doi:10.1212/WNL.0000000000009764

21. Goyal M, Menon BK, van Zwam WH, et al. Endovascular thrombectomy after large-vessel ischaemic stroke: a meta-analysis of individual patient data from five randomised trials. Lancet. 2016;387(10029):1723-1731. doi:10.1016/S0140-6736(16)00163-X

22. Regenhardt RW, Young MJ, Etherton MR, et al. Toward a more inclusive paradigm: thrombectomy for stroke patients with pre-existing disabilities. J Neurolntervent Surg. Published online October 30, 2020:neurintsurg-2020-016783. doi:10.1136/neurintsurg-2020-016783

23. Gorelick Philip B., Furie Karen L., ladecola Costantino, et al. Defining Optimal Brain Health in Adults: A Presidential Advisory From the American Heart Association/American Stroke Association. Stroke. 2017;48(10):e284-e303. doi:10.1161/STR.0000000000000148

24. Franke K, Gaser C, Manor B, Novak V. Advanced BrainAGE in older adults with type 2 diabetes mellitus. Front Aging Neurosci. 2013;5:90-90. doi:10.3389/fnagi.2013.00090

25. Regenhardt RW, Takase H, Lo EH, Lin DJ. Translating concepts of neural repair after stroke: Structural and functional targets for recovery. Restor Neurol Neurosci. 2020;38(1):67-92. doi:10.3233/RNN-190978

26. Bretzner M, Bonkhoff AK, Schirmer MD, et al. MRI Radiomic Signature of White Matter Hyperintensities Is Associated With Clinical Phenotypes. Front Neurosci. 2021;15:691244. doi:10.3389/fnins.2021.691244

27. Peng H, Gong W, Beckmann CF, Vedaldi A, Smith SM. Accurate brain age prediction with lightweight deep neural networks. Medical Image Analysis. 2021;68:101871. doi:10.1016/j.media.2020.101871

28. Liem F, Varoquaux G, Kynast J, et al. Predicting brain-age from multimodal imaging data captures cognitive impairment. Neurolmage. 2017;148:179-188. doi:10.1016/j.neuroimage.2016.11.005

29. Cole JH, Poudel RPK, Tsagkrasoulis D, et al. Predicting brain age with deep learning from raw imaging data results in a reliable and heritable biomarker. Neurolmage. 2017;163:115-124. doi:10.1016/j.neuroimage.2017.07.059

\section{Figures}



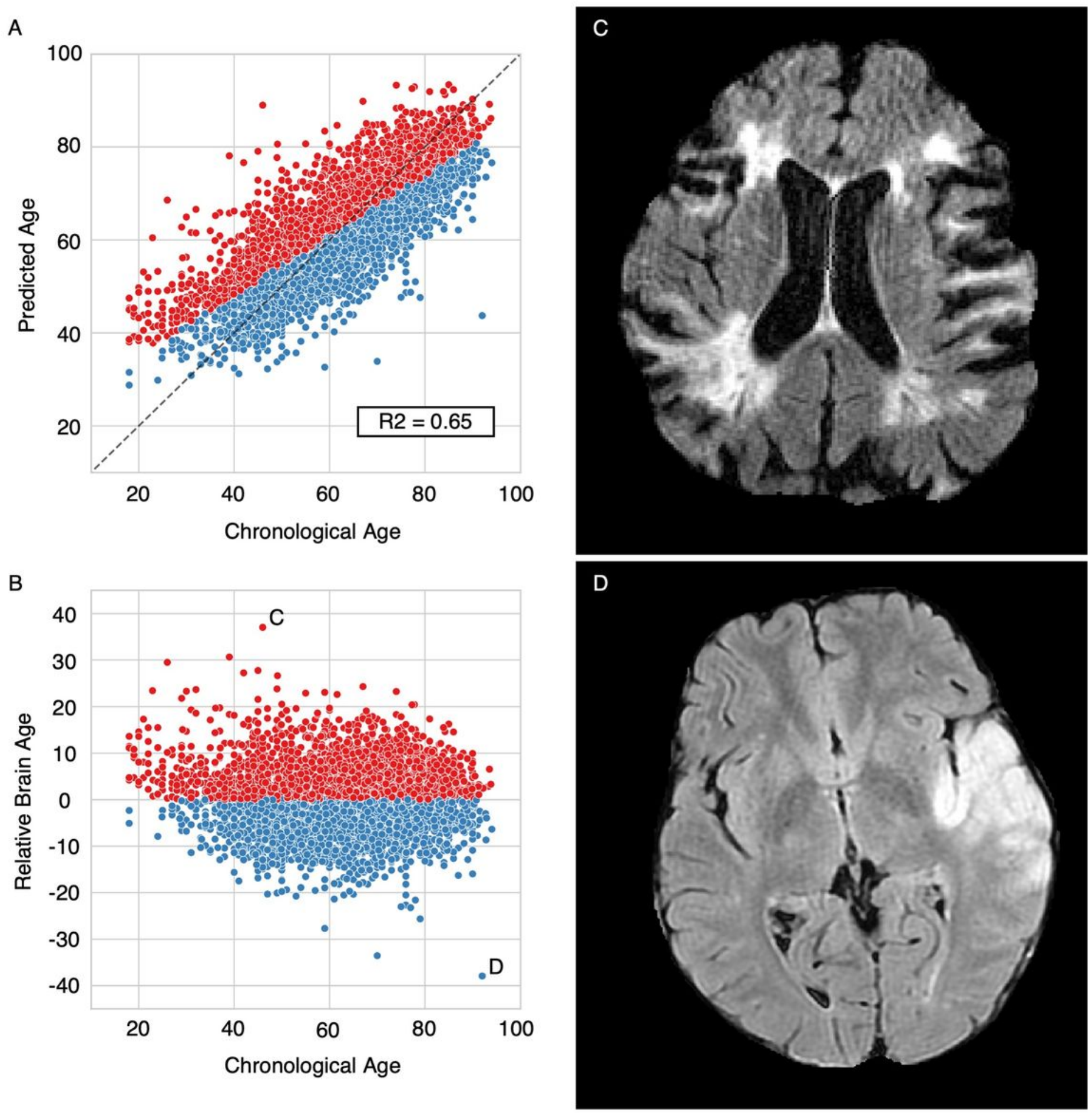

Figure 1

Brain age prediction performances and relative brain age. 


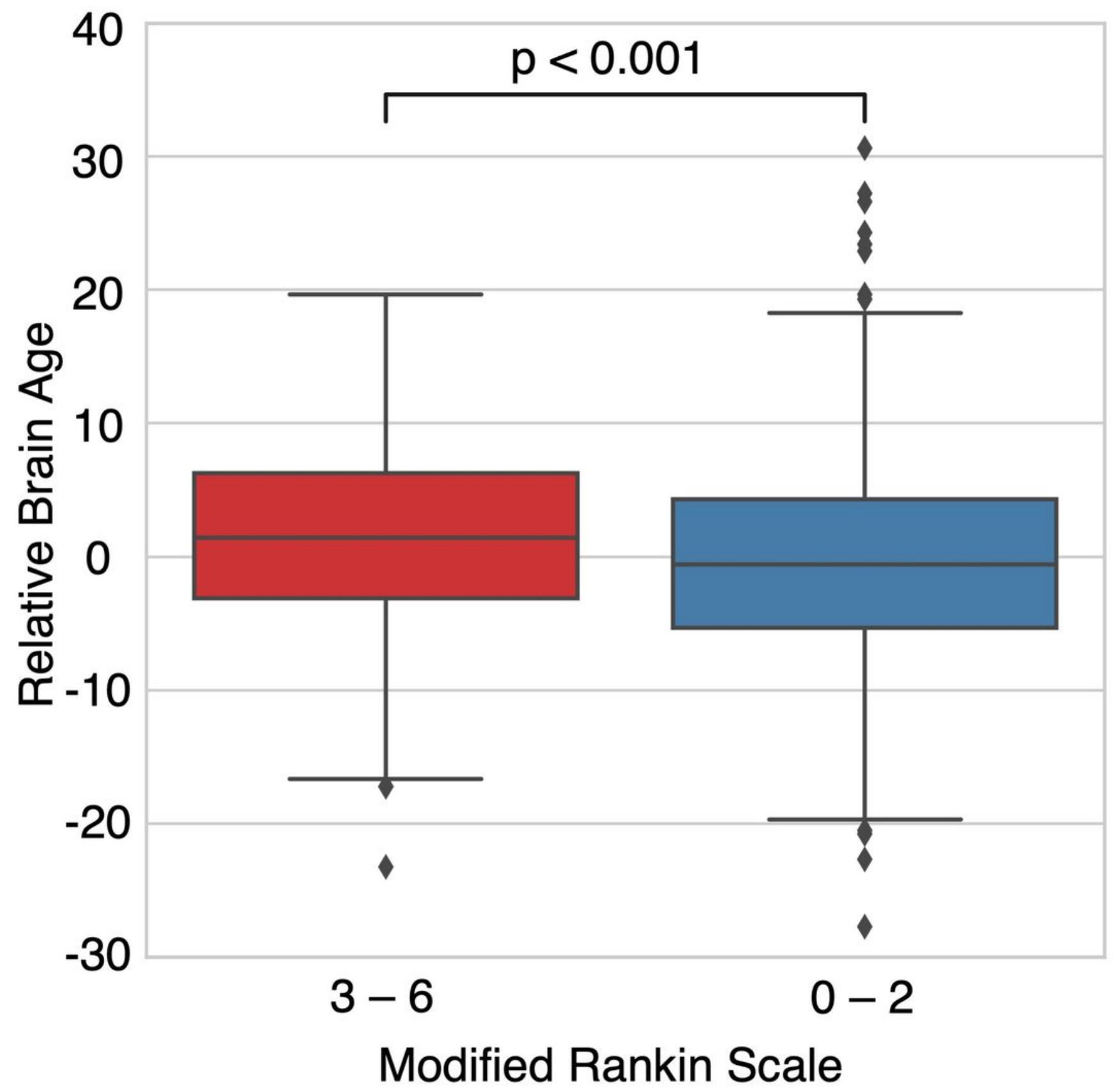

Figure 2

Boxplot of RBA per outcome group 


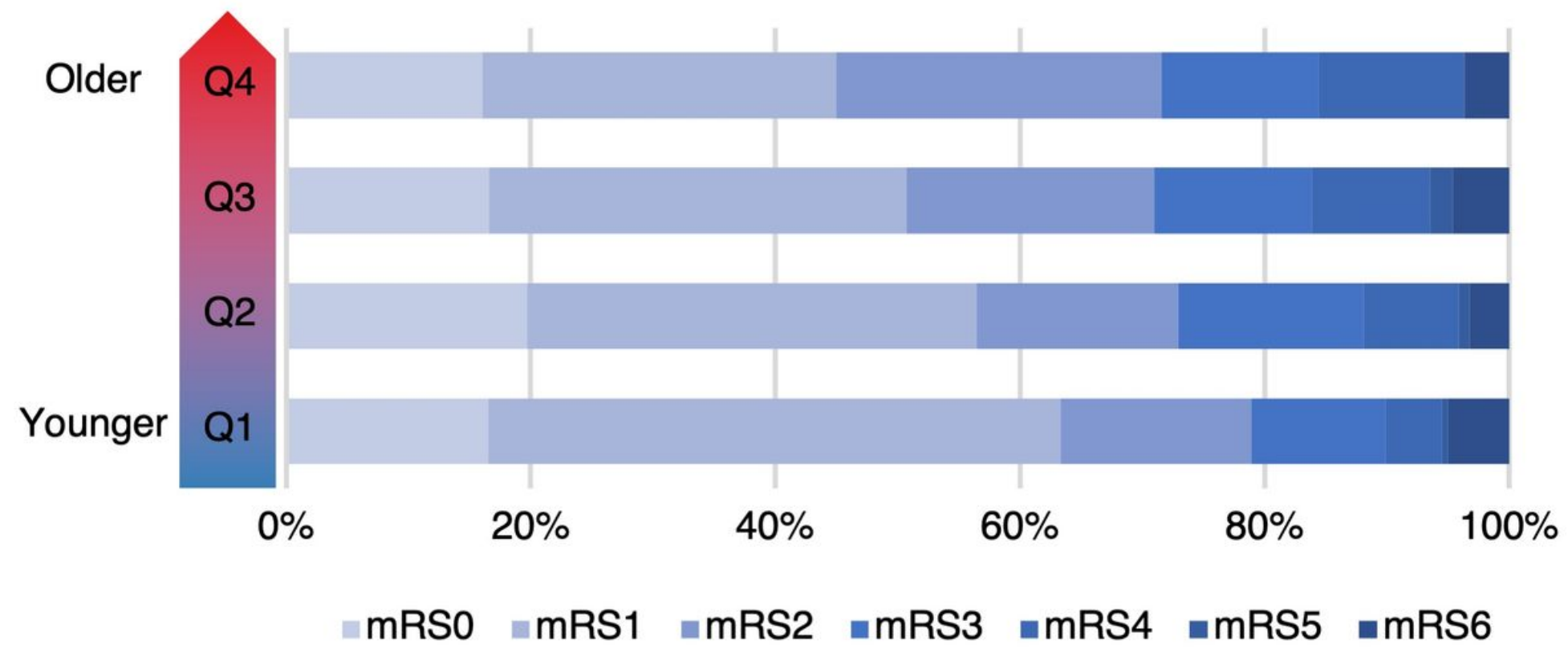

Figure 3

mRS shift plot per quartile of relative brain age. 


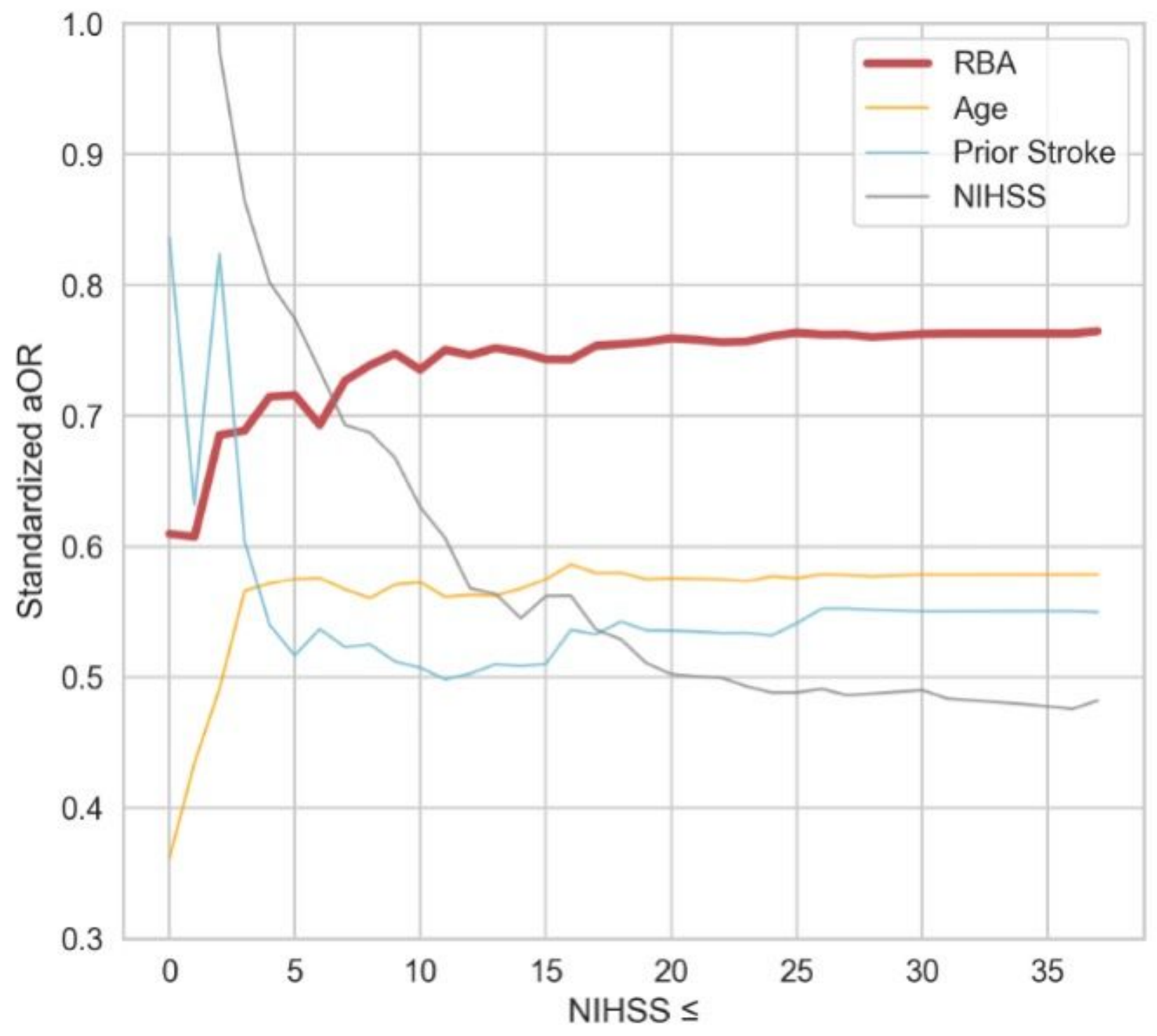

Figure 4

Effect sizes of the predictors of good functional outcome by rank of baseline NIHSS. 